\title{
MiR-382-5p controls hematopoietic stem cell differentiation through the downregulation of MXD1
}

Roberta Zini ${ }^{1}$, Chiara Rossi ${ }^{1}$, Ruggiero Norfo ${ }^{1,2}$, Valentina Pennucci ${ }^{1}$, Greta Barbieri ${ }^{1}$, Samantha Ruberti ${ }^{1}$, Sebastiano Rontauroli ${ }^{1}$, Simona Salati ${ }^{1}$, Elisa Bianchi ${ }^{1}$, and Rossella Manfredini ${ }^{1}$

${ }^{1}$ Centre for Regenerative Medicine, Department of Life Sciences, University of Modena and Reggio Emilia, Modena, Italy; ${ }^{2}$ Haematopoietic Stem Cell Biology Laboratory, Weatherall Institute of Molecular Medicine, University of Oxford, Oxford, United Kingdom.

RUNNING TITLE: Role of miR-382/MXD1 axis in hematopoietic stem cell fate CORRESPONDING AUTHORS: Rossella Manfredini, PhD, Centre for Regenerative Medicine "Stefano Ferrari", University of Modena and Reggio Emilia, via Gottardi n.100, 41125 Modena, Italy. E-mail: rossella.manfredini@unimore.it Roberta Zini, PhD, Centre for Regenerative Medicine "Stefano Ferrari", University of Modena and Reggio Emilia, via Gottardi n.100, 41125 Modena, Italy. E-mail: roberta.zini@unimore.it

FOOTNOTES AND ABBREVIATIONS: m-NegCTR, miRNA mimic Negative Control; ss-NegCTR; siRNA Negative Control. 
ROBERTA ZINI, roberta.zini@unimore.it

Centre for Regenerative Medicine, Department of Life Sciences, University of Modena and Reggio Emilia, via Gottardi n.100, 41125 Modena, Italy. Phone +390592058297 Fax +390592058115

CHIARA ROSSI, 204019@studenti.unimore.it

Centre for Regenerative Medicine, Department of Life Sciences, University of Modena and Reggio Emilia, via Gottardi n.100, 41125 Modena, Italy. Phone +390592058061 Fax +390592058115

RUGGIERO NORFO, ruggiero.norfo@gmail.com

Centre for Regenerative Medicine, Department of Life Sciences, University of Modena and Reggio Emilia, via Gottardi n.100, 41125 Modena, Italy. Phone +390592058061 Fax +390592058115

VALENTINA PENNUCCI, valentinapennucci@alice.it

Centre for Regenerative Medicine, Department of Life Sciences, University of Modena and Reggio Emilia, via Gottardi n.100, 41125 Modena, Italy. Phone +390592058061 Fax +390592058115

GRETA BARBIERI, 165682@studenti.unimore.it

Centre for Regenerative Medicine, Department of Life Sciences, University of Modena and Reggio Emilia, via Gottardi n.100, 41125 Modena, Italy. Phone +390592058061 Fax +390592058115

SAMANTHA RUBERTI, samantha.ruberti@unimore.it

Centre for Regenerative Medicine, Department of Life Sciences, University of Modena and Reggio Emilia, via Gottardi n.100, 41125 Modena, Italy. Phone +390592058061 Fax +390592058115 
SEBASTIANO RONTAUROLI, 78087@studenti.unimore.it

Centre for Regenerative Medicine, Department of Life Sciences, University of Modena and Reggio Emilia, via Gottardi n.100, 41125 Modena, Italy. Phone +390592058061 Fax +390592058115

SIMONA SALATI, simona.salati@unimore.it

Centre for Regenerative Medicine, Department of Life Sciences, University of Modena and Reggio Emilia, via Gottardi n.100, 41125 Modena, Italy. Phone +390592058297 Fax +390592058115

ELISA BIANCHI, elisa.bianchi@unimore.it

Centre for Regenerative Medicine, Department of Life Sciences, University of Modena and Reggio Emilia, via Gottardi n.100, 41125 Modena, Italy. Phone +390592058307 Fax +390592058115

ROSSELLA MANFREDINI, rossella.manfredini@unimore.it

Centre for Regenerative Medicine, Department of Life Sciences, University of Modena and Reggio Emilia, via Gottardi n.100, 41125 Modena, Italy. Phone +390592058065 Fax +390592058115 


\begin{abstract}
MicroRNAs are key regulators of gene expression that control stem cell fate by posttranscriptional down-regulation of hundreds of target genes via seed-pairing in their 3' untranslated region. In fact, miRNAs tightly regulate fundamental stem cell processes, like self-renewal, proliferation and differentiation; therefore, miRNA deregulation may contribute to the development of solid tumours and haematological malignancies.
\end{abstract}

MiR-382-5p has been found to be upregulated in patients with myeloid neoplasms but its role in normal hematopoiesis is still unknown. Here we demonstrated that miR382-5p overexpression in CD34+ hematopoietic stem/progenitor cells leads to a significant decrease of megakaryocyte precursors coupled to increase of granulocyte ones. Furthermore, by means of a computational analysis using different prediction algorithms, we identified several putative mRNA targets of miR-382-5p that are downregulated upon miRNA overexpression (i.e. FLI1, GATA2, MAF, MXD1, RUNX1 and SGK1). Among these, we validated MXD1 as real target of miR-382-5p by luciferase reporter assay. Finally, we showed that MXD1 knockdown mimics the effects of miR-382-5p overexpression on granulocyte and megakaryocyte differentiation of CD34+ cells.

Overall, our results demonstrated that miR-382-5p expression favours the expansion of granulocyte lineage and impairs megakaryocyte commitment through MXD1 downregulation. Therefore, our data showed for the first time that the miR-382-5p/MXD1 axis plays a critical role in myelopoiesis by affecting the lineage choice of CD34+ hematopoietic stem/progenitor cells. 


\section{INTRODUCTION}

MicroRNAs (miRNAs) are a large class of small noncoding RNAs that act as posttranscriptional regulators of gene expression (1). miRNAs simultaneously modulate the expression of several targets by binding to the 3 '-untraslated region (3'-UTR) of the mRNAs, resulting in mRNA destabilization and/or translational inhibition (2). In the last decade, growing evidence has unravelled the role of miRNAs as key regulators of stem cell fate by modulating essential processes like self-renewal, proliferation and differentiation. In particular, the combinatorial expression of miRNAs can precisely characterize specific cell types and regulate different phases of differentiation $(3,4)$. Moreover, recent advancements in computational and molecular biology have highlighted the existence of regulatory networks where miRNAs modulate the expression of transcription factors and transcription factors regulate miRNA levels to promote or maintain some important cell functions (1). On the other side, miRNA expression deregulation may contribute to the development of different human diseases, including cancer (5).

In this study, we focused our attention on miR-382-5p, which resulted to be deregulated in several solid tumours and hematologic neoplasms (6-9). In particular, miR-382-5p upregulation has been reported in Acute Promyelocytic Myeloid Leukemia (AML-M3) $(10,11)$ and in Primary Myelofibrosis (PMF) patients (12). Despite an abnormal expression level of miR-382-5p has been described in these hematological malignancies, its role in normal hematopoiesis has not been described so far. Therefore, in order to characterize the function of miR-382-5p in myeloid development, we evaluated the effects of its overexpression on the commitment of human CD34+ hematopoietic stem/progenitor cells (HSPCs). Interestingly, we identified MAX dimerization protein 1 (MXD1) as a target of miR-382-5p, and 
demonstrated that miR-382-5p favours granulocyte lineage at the expense of megakaryocyte one through MXD1 downregulation.

\section{MATERIALS AND METHODS \\ Human CD34+ cells purification}

Umbilical cord blood (CB) samples were collected after normal deliveries, according to the institutional guidelines for discarded material (Clearance of Ethical Commitee for Human experimentation of Modena: Secretary office Saverio Santachiara, santachiara.saverio@policlinico.mo.it, approval date: 18.01.2005; approval file number \# 793/CE). CB CD34+ cells were purified as previously described (13). After immunomagnetic separation, CD34+ cells were seeded in 24-well plates at $5 \times$ $10^{5} / \mathrm{ml}$ in Iscove's modified Dulbecco's medium (IMDM) (Euroclone SPA, Milan, Italy) containing 20\% human serum (Bio-Whittaker, Walkersville, MD, USA), stem cell factor (SCF) $(50 \mathrm{ng} / \mathrm{ml})$, FLT3-ligand (FLT3L) $(50 \mathrm{ng} / \mathrm{ml})$, thrombopoietin (TPO) (20 ng/ml), interleukin-6 (IL6) (10 ng/ml), and interleukin-3 (IL3) (10 ng/ml) (all from Miltenyi Biotec, Auburn, CA, USA).

\section{Electroporation of CD34+ cells}

Human CD34+ cells were transfected by using the 4D-Nucleofector System (Lonza Group Ltd, Basel, Switzerland) as previously reported (12). Briefly, after 24 hours of incubation in the cell-culture condition described above, CD34+ cells were nucleofected twice, once every 24 hours, with mirVana miR-382-5p mimic or mirVana miRNA mimic Negative Control \#1 (m-NegCTR) (Life Technologies, Carlsbad, CA, USA). For each electroporation, $4 \times 10^{5} \mathrm{CD} 34+$ cells were resuspended in $100 \mu \mathrm{L}$ of P3 Primary Cell Solution (Lonza) containing $3 \mu \mathrm{g}$ of mimic miRNA, and pulsed with 
the program DS112. After each transfection, CD34+ cells were transferred into prewarmed fresh medium in 24-well plates (Euroclone) and maintained in the same culture conditions as described above.

For small interfering RNAs (siRNAs) transfection, each sample was electroporated 3 times, once every 24 hours, with $3 \mu \mathrm{g}$ of a mix of 3 Silencer Select siRNA targeting human MXD1 (supplemental Table 1) (Life Technologies). As previously described, CD34+ cells were nucleofected 24 hours after immunomagnetic purification, by using the P3 Primary Cell Solution (Lonza) and the electroporation protocol DS112. To exclude nonspecific effects caused by interfering RNA (RNAi) nucleofection, a sample transfected with a non-targeting siRNA negative control (ss-NegCTR; Silencer Select Negative Control \#2 siRNA; Life Technologies) was included.

Cells were analyzed 24 and 48 hours after the last nucleofection for both cell viability and miR-382-5p and/or MXD1 expression.

Viability measurement was assessed by trypan blue exclusion assay 24 hours after the last nucleofection (14). In a Neubauer chamber, at least 100 cells were microscopically analyzed in duplicate for viability. The mean percentage of living cells of the two analyses was calculated.

\section{CD34+ cell-culture conditions, morphological and immunophenotypic analysis}

CD34+ cells were plated $\left(5 \times 10^{5} / \mathrm{mL}\right)$ in IMDM with the addition of $20 \%$ BIT 9500 serum substitute (bovine serum albumin, insulin, and transferrin; StemCell Technologies) 24 hours after the last nucleofection, in order to set up a multilineage cell culture (SCF, 50 ng/mL; FLT3L, 50 ng/mL; TPO, 20 ng/mL; IL-3, 10 ng/mL; IL-6, $10 \mathrm{ng} / \mathrm{mL}$; all cytokines from Miltenyi), megakaryocyte (MK) unilineage culture (TPO, 
$100 \mathrm{ng} / \mathrm{mL}$ ), and granulocyte (GN) unilineage culture (SCF, $10 \mathrm{ng} / \mathrm{mL}$; G-CSF, 25 $\mathrm{ng} / \mathrm{mL})(13)$.

Differentiation of CD34+ cells was monitored by morphological analysis of MayGrunwald-Giemsa-stained cytospins and by flow cytometry analysis of CD34, CD14, CD66b, CD15, Glycophorin A (GPA), CD41 and CD42b surface antigen expression at day $5,7,10$, and 12 after the last nucleofection. Images were captured by using an Ax10scopeA1 microscope equipped with AxioCam ERc 5S Digital Camera and Axion software 4.8 (all Carl Zeiss Microlmaging Inc.; Thornwood, NY, USA). The images were then processed with Adobe Photoshop 11.0.2 software.

The following monoclonal antibodies (MoAbs) were used for flow cytometry analysis: phycoerythrin (PE)-conjugated mouse anti-human CD14 MoAb, fluorescein isothiocyanate (FITC)-conjugated mouse anti-human CD34 MoAb, FITC-conjugated mouse anti-human CD66b MoAb, FITC-conjugated mouse anti-human CD15 MoAb (all from Miltenyi Biotech; Auburn, CA, USA), FITC-conjugated mouse anti- human CD41 MoAb, PE-conjugated mouse anti-human CD42b MoAb, and PE-conjugated mouse anti-human GPA MoAb (all from Dako; Milano, Italia). After staining, cells were analyzed by using a BD FACSCanto II (BD Biosciences; San Jose, CA USA). At least 10,000 events were counted for each sample to ensure statistical relevance.

MiR-382-5p expression during erythroid, megakaryocyte, granulocyte and mono-macrophage differentiation

After immunomagnetic separation, CD34+ cells were seeded in 24-well plate at a density of $5 \times 10^{5} / \mathrm{ml}$ in IMDM (Euroclone SPA, Milan, Italy) containing $20 \%$ human serum (Bio-Whittaker, Walkersville, MD, USA), SCF $50 \mathrm{ng} / \mathrm{ml}$, FLT3L $50 \mathrm{ng} / \mathrm{ml}$, TPO $20 \mathrm{ng} / \mathrm{ml}$, IL6 $10 \mathrm{ng} / \mathrm{ml}$, and IL3 $10 \mathrm{ng} / \mathrm{ml}$ (all from Miltenyi Biotec, Auburn, 
CA, USA). After a first phase of expansion, at 24 hours of culture (day 0 ) cells were seeded $(5 \times 105 / \mathrm{ml})$ in IMDM added with $20 \%$ BIT (StemCell Technologies) in order to set up erythrocyte (EPO 0,4U/ml, SCF $10 \mathrm{ng} / \mathrm{ml}$ ), megakaryocyte (TPO 100 $\mathrm{ng} / \mathrm{ml}$ ), granulocyte (GCSF $25 \mathrm{ng} / \mathrm{ml}$, SCF $10 \mathrm{ng} / \mathrm{ml}$ ) and monocyte (MCSF 100 ng/ml, SCF $20 \mathrm{ng} / \mathrm{ml}$, IL6 $20 \mathrm{ng} / \mathrm{ml}$ and Flt3L $50 \mathrm{ng} / \mathrm{ml}$ ) unilineage cultures (13). CD34+ cells differentiation was monitored by morphological analysis of MGG-stained cytospins and by flow-cytometric analysis of differentiation marker expression. The medium was replaced every 2 days. MiR-382-5p expression level was detected by qRT-PCR at different time points (i.e. days 1, 2, 3, 5, 7, 9 and 12) after seeding cells in erythrocyte, megakaryocyte, granulocyte or mono-macrophage unilineage cultures. The day 0 sample was designated as the calibrator.

\section{Methylcellulose and collagen clonogenic assays}

Two different clonogenic assays were performed at 24 hours after the last nucleofection. The methylcellulose assay was carried out by plating CD34+ cells in MethoCult ${ }^{\mathrm{TM}} \mathrm{H} 4434$ Classic (StemCell Technologies Inc.; Vancouver), as previously described (15). In addition, MK colony forming units (CFU-MK) were assayed in collagen-based medium, using a commercial MK assay detection kit (MegaCult-C; StemCell Technologies Inc.) as previously reported (16).

\section{RNA purification and quantitative reverse transcription polymerase chain reaction (qRT-PCR)}

Total cellular RNA, including small RNAs, was isolated from CD34+ cells using the miRNeasy mini RNA isolation kit (Qiagen, Hilden, Germany) following the manufacturer's recommendations. The purity and integrity of RNA samples were 
determined by using disposable RNA chips (Agilent RNA 6000 Nano LabChip kit) and the Agilent 2100 Bioanalyzer (Agilent Technologies; Waldbrunn, Germany). NanoDrop ND-1000 spectrophotometer (NanoDrop Technologies; Wilmington, DE) was used to evaluate the RNA sample concentration, while $260 / 280 \mathrm{~nm}$ and $260 / 230$ $\mathrm{nm}$ ratios were used to assess the purity of RNA.

cDNA was reverse-transcribed from total RNA (100 ng per sample) using the HighCapacity cDNA Reverse Transcription Kit (Life technologies; Carlsbad, CA, USA), and real-time PCR was carried out in triplicate with TaqMan gene expression assays and Fast advanced master mix (all reagents from Life Technologies) by using an $A B$ 7900HT Fast Real-Time PCR System (Applied Biosystems) (17). Gene expression relative quantification (RQ) was achieved using the comparative cycle threshold (CT) method using glyceraldehyde-3-phosphate dehydrogenase (GAPDH) as the housekeeping gene. The ss-NegCTR sample was considered as the calibrator, the $R Q$ value was expressed as $2-\Delta \Delta C T$.

For miRNA cDNA synthesis, $5 \mathrm{ng}$ of total RNA was reverse-transcribed using the Taqman microRNA Reverse Transcription Kit and specific primers included in TaqMan MicroRNA assays. qRT-PCR was performed using TaqMan MicroRNA assays (Life Technologies) as described above and U6 snRNA as a housekeeping control.

\section{Luciferase reporter assays}

Predicted target sites of miR-382-5p were cloned into the Pme1 and Xbal sites of pmirGLO Dual-Luciferase miRNA Target Expression Vector (Promega Italia, Milano, Italy), following manufacturer's instructions. Every cloned plasmid contains the firefly luciferase gene upstream of a given miRNA binding site, and the Renilla luciferase 
gene acting as a normalizer gene. Annealing oligonucleotides containing wild type miRNA binding sites were designed based on TargetScan 7.0 sequences (Supplemental Table 2). Mutated miRNA binding sites were obtained from the wildtype miRNA binding sites by changing nucleotides 3-5 in the miRNA seed regions. K562 cells were electroporated by means of the Amaxa 4D-NucleofectorTM System, according to the manufacturer's instructions. Briefly, K562 cells were cultured at a density of $3 \times 10^{5}$ cells $/ \mathrm{mL} 2$ days before nucleofection. Cells were then transiently conucleofected with either a miR-382-5p or m-NegCTR at a concentration of $3.6 \mu \mathrm{M}$ and with either empty vector or miRNA binding site-containing construct at a concentration of $200 \mathrm{ng} / \mathrm{sample}$. For each electroporation, $1 \times 10^{6}$ cells were resuspended in $100 \mu \mathrm{L}$ of SF Cell line Solution (Lonza) and pulsed with the program FF120. Firefly and Renilla luciferase activities were measured 24 hours after electroporation using the Dual-Luciferase Reporter Assay System (Promega), and luminescence was recorded on a GloMax®-Multi+ Detection System with Intinct ${ }^{\mathrm{TM}}$ Software (Promega), according to the manufacturer's protocol.

In order to analyze the data, three levels of normalization were performed as previously described: firstly, firefly luciferase activity was normalized to that of Renilla luciferase as the internal control for nucleofection efficiency; secondly, the data were normalized to the effect of the miRNA mimic on the empty vector control reporter; lastly, the luminescence signals were normalized to the effect of the m-NegCTR on the corresponding miRNA binding site-containing construct (12).

\section{Western blot}

MXD1 protein level in both miR-382-5p-overexpressing cells and MXD1-siRNA transfected CD34+ cells was detected by Western blot analysis. Briefly, cells were 
harvested, washed twice with ice-cold PBS and lysed $\left(5 \times 10^{5}\right.$ cells $/ 50 \mu$ l of lysis buffer) in $50 \mathrm{mM}$ Tris (tris(hydroxymethyl)aminomethane) $-\mathrm{Cl}(\mathrm{pH} 7.4), 150 \mathrm{mM} \mathrm{NaCl}$, $10 \mathrm{mM} \mathrm{KCl}, 1 \mathrm{mM}$ EDTA, $20 \mathrm{mM} \mathrm{NaF}, 0.25 \% \mathrm{Na}$ doexycholate, $5 \mathrm{mM}$ dithiothreitol (DTT), 1\% Nonidet P-40, and protease inhibitors (Complete, catalog \#1697498, Roche, Milano, Italy). Total cell lysates (30 $\mu \mathrm{g}$ for each sample) were loaded onto $10 \%$ SDS-polyacrylamide gel and blotted on nitrocellulose membrane. The membranes were stained with Red Ponceau to control loading and transfer. Membranes were then pre-blocked in blocking solution, composed by $5 \%$ bovine serum albumin (BSA) in $0.1 \%$ TBST, for 1 hour at room temperature (RT), and incubated with primary antibodies: 1) rabbit polyclonal anti-MXD1 antibody (1:500 dilution, anti-Mad1 (C19), catalog \#sc-222, Santa Cruz Biotechnology Inc., Heidelberg, Germany) at $4{ }^{\circ} \mathrm{C}$ overnight; 2) rabbit polyclonal anti-actin antibody (1:2000 dilution, Thermo Fisher Scientific Inc., Waltham, MA, USA, catalog \#PA116889) for 1 hour at RT. After three washes with TBST, blots were incubated with HRP conjugated goat anti-rabbit secondary antibody (1:10000 dilution, catalog \#sc2004, Santa Cruz Biotechnology, Inc.) for 1 hour at RT and revealed by BM Chemiluminescence Blotting Substrate (POD) (Roche).

\section{Statistical analysis}

The statistics used for data analysis in overexpression/silencing experiments and luciferase reporter assays were based on 2-tailed Student $t$ tests for averages comparison in paired samples. Data were analyzed by using Microsoft Excel (Microsoft Office, 2008 release) and are reported as mean \pm standard error of the mean (SEM). $\mathrm{P}<.05$ was considered significant. 


\section{RESULTS}

\section{MiR-382-5p overexpression in CD34+ cells}

In order to study the role of miR-382-5p in HSPC commitment, miR-382-5p was overexpressed in CD34+ cells. By means of qRT-PCR, we confirmed that the miRNA expression level was upregulated upon miR-382-5p mimic transfection at 24 and 48 hours (RQ \pm SEM, $1677 \pm 383, \mathrm{P}<.05$ and $625 \pm 85 \mathrm{P}<.01$, respectively) after the last nucleofection (Figure 1A). Trypan blue exclusion assay showed that cell viability was about $90 \%$ in both $\mathrm{m}-\mathrm{NegCTR}$ and miR-382-5p samples (Figure $\mathbf{S 1 A}$ ).

Flow cytometry analysis of MK differentiation markers performed on TPO unilineage culture at days $5,7,10$, and 12 showed that miR-382-5p overexpression induces a significant decrease in the CD41+ cell fraction compared with the m-NegCTR control (Figure 1B). In addition, the mature megakaryocytic CD41+/CD42b+ population was similarly reduced at days 7 and 10 (Figure 1C). On the contrary, miR-382-5p overexpression in GCSF unilineage culture led to a significant increase in the percentage of $\mathrm{CD} 66 \mathrm{~b}+$ and $\mathrm{CD} 15+$ cells at days 10 and 12 after the last nucleofection (Figure 1D-E). Similarly, miR-382-5p upregulation in multilineage culture conditions further confirmed these results (data not shown). Flow cytometry analysis of mono-macrophage and erythrocyte differentiation markers did not highlight any significant modulation of expression between miR-382-5p CD34+ cells and the m-NegCTR sample (data not shown). According to flow cytometry results, the morphologic analysis of MGG-stained cytospins obtained from TPO unilineage culture showed a remarkable reduction in MK precursors at different stages of maturation in miR-382-5p-overexpressing cells compared with control (Figure 1G, Panels i-iv). Moreover, morphological evaluation of GCSF-treated cells clearly displayed a considerable enrichment in granulocytes in miR-382-5p sample (Figure 
1H, Panels i-iv). Similarly, miR-382-5p upregulation in multilineage culture conditions further confirmed these results (Figure 2). Flow cytometry analysis of monomacrophage (CD14) and erythrocyte (GPA) markers did not highlight any significant modulation of expression between miR-382-5p CD34+ cells and the m-NegCTR sample (Figure S1, Panels B-D).

Consistently with previous results, methylcellulose assay highlighted a remarkable increase in the percentage of granulocyte colony forming unit (CFU-G), whereas monocyte (CFU-M), erythroid (Burst-Forming Units (BFU-E) and CFU-E) and granulo-monocyte (CFU-GM) colonies were not significantly affected (Figure 3A). Furthermore, the collagen-based assay that supports the growth of MK progenitors in vitro showed that miR-382-5p overexpression causes a significant decrease in the CFU-MK percentage coupled with a significant increase of non-MK colonies (Figure 3B).

Since miR-382-5p interferes with lineage choice of CD34+ cells, we assessed its expression level in CD34+ and differentiated cells obtained from granulocyte, monocyte-macrophage, erythrocyte, and megakaryocyte unilineage culture conditions. As shown in figure S2, miR-382-5p expression level was decreased during the initial phase of megakaryocyte and erythroid unilineage cultures, while its expression increased in the last phases of differentiation. On the contrary, miR-382$5 p$ results upregulated during the granulocyte and monocyte-macrophage differentiation (Figure S2).

Overall, these results indicated that miR-382-5p overexpression in CD34+ HSPCs enhances granulocyte differentiation while negatively interferes with the megakaryocyte one. 


\section{MiR-382-5p target prediction and validation by luciferase reporter assays}

In order to identify the molecular targets through which miR-382-5p modulates the MK and GN commitment in CD34+ HSPCs, several prediction algorithms (i.e. TargetScan, microRNA.org, PITA, etc.) were used. In a combinational analysis with these different tools we found out several predicted targets of miR-382-5p, which contain a putative binding site for miR-382-5p in their $3^{\prime}-$ UTR. Next, we selected those genes known to be involved in MK and GN differentiation (i.e. FLI1, GATA2, MAF, MXD1, RUNX1, and SGK1) (Table 1) (18-22).

First, we assessed whether miR-382-5p overexpression in CD34+ HSPCs led to the down-regulation of putative target expression by means of qRT-PCR. As shown in Figure $4 \mathrm{~A}$, all mRNAs were downregulated in miR-382-5p-overexpressing cells at 24 hours after the last nucleofection.

Finally, to confirm whether the downregulated mRNAs are real targets of miR-382-5p, we validated every putative miRNA-mRNA interaction by means of luciferase reporter assay. To this end, the predicted miRNA binding sites were cloned into the 3'-UTR of the Dual Luciferase pmirGLO vector and transiently transfected in K562 cells. Luciferase activity was measured in the presence of miR-382-5p mimic or $\mathrm{m}$ NegCTR and normalized using Renilla activity. As shown in Figure 4B, miR-382-5p overexpression significantly inhibited the luciferase activity in the sample transfected with the wild type miRNA binding site on MXD1 3'-UTR; conversely, the luciferase activity was not repressed by miR-382-5p overexpression in sample transduced with MXD1-mutated miRNA binding site. Likewise, miR-382-5p upregulation did not significantly reduce the luciferase activity in samples transduced with miRNA binding site of GATA2, FLI1, RUNX1, MAF and SGK1 (Figure 4B). According to luciferase 
reporter assay results, Western blot analysis showed a marked reduction of MXD1 protein level upon miR-382-5p overexpression (Figure 4C).

\section{MXD1 silencing in CD34+ cells}

To better characterize the role of miR-382-5p/MXD1 axis in myelopoiesis, we investigated whether MXD1 silencing could reproduce the effects mediated by miR382-5p overexpression on GN and MK differentiation. Therefore, CD34+ cells were transfected with a mixture of three Silencer Select siRNAs targeting MXD1 mRNA (supplemental Table 1) and with a non-targeting siRNA as a negative control (ssNegCTR). As shown in Figure S3 Panel A, cell viability was $>90 \%$ in all samples, without any significant differences between ss-NegCTR and MXD1-siRNA. The expression level of MXD1 in control samples and MXD1-siRNA cells was assessed by qRT-PCR and Western Blot analysis. As shown in Figure 5A, we obtained a significant MXD1 downregulation of MXD1 mRNA at 24 and 48 hours after the last nucleofection $(R Q \pm S E M, 0.34 \pm 0.03, P<.001$ and $0.48 \pm 0.10, P<.01$, respectively). Accordingly, the MXD1 protein level is remarkably decreased at 24 hours post-nucleofection (Figure 5B).

As observed for the miR-382-5p overexpression, MXD1 silencing led to a substantial decrease in the percentage of CD41+ and CD41+/CD42b+ cells at day 7 and 10 after the last nucleofection in MK unilineage culture (Figure 5C-D). At the same time, MXD1 silencing induces a significant increase in the percentage of CD66b+ and CD15+ cells compared with the ss-NegCTR sample (Figure 5E-F). Furthermore, the morphological analysis of MGG-stained cytospins showed a significant reduction in MK precursors (Figure $\mathbf{5 H}$, Panels i-iv) and a significant expansion of granulocytes in MXD1-siRNA cells (Figure 5l, Panels i-iv). The same morphological differences 
could be appreciated also in MGG-stained cytospins obtained from multilineage culture (Figure 5J, Panels i-iv).

Conversely, we did not observe any modulation of GPA and CD14 expression upon MXD1 knockdown (Figure S3, Panels B-D).

Finally, according to results obtained by means of clonogenic assay on miR-382-5poverexpressing cells, we found a significant increase in the percentage of CFU-G, while CFU-M, BFU-E/CFU-E and CFU-GM colonies were not significantly affected by MXD1 down-regulation (Figure 6A). In addition, the collagen-based assay confirmed that MXD1 silencing induces a decrease of MK progenitors as showed by a lower CFU-MK percentage in MXD1-siRNA sample compared with control (Figure 6B).

Since the expression levels of the megakaryocyte transcription factors GATA2, FLI1, RUNX1, and MAF and of the kinase SGK1 were decreased upon miR-382-5p overexpression, although they have not been previously identified as functional targets, we still wondered whether MXD1 silencing could modulate their expression. By means of qRT-PCR, we showed that the mRNA levels of GATA2, RUNX1 and FLI1 were significantly downregulated in MXD1-silenced cells (Figure 7). Conversely, MAF and SGK1 expression levels seem not to be affected by MXD1 knockdown.

\section{DISCUSSION}

Growing evidence shows that miRNAs regulate stem cell-fate decision by modulating fundamental processes like self-renewal, proliferation and differentiation. For this reason, dysregulation of miRNA expression represents a common mechanism of tumorigenesis and cancer progression. 
MiR-382-5p overexpression has been described in some hematological malignancies, such as PMF (12) and Acute Myeloid Leukemia (AML) $(10,11)$, even if its role in normal hematopoiesis was still unknown.

Here we showed for the first time that miR-382-5p plays a critical role in hematopoiesis by influencing the lineage choice of CD34+ cells. In particular, we demonstrated that miR-382-5p supports the expansion of granulocyte lineage whereas negatively interferes with megakaryocyte commitment, as demonstrated by a significant increase of granulocyte precursors coupled to decrease of megakaryocyte ones in miR-382-5p-overexpressing cells (Figure 1-3).

Next, in order to identify the molecular targets by which miR-382-5p regulates HSPC fate, we found by a prediction analysis several putative targets known to be involved in MK and GN differentiation (i.e. FLI1, GATA2, MAF, MXD1, RUNX1, and SGK1) (Table 1) (18-22), whose mRNA expression was decreased in miR-382-5poverexpressing cells (Figure 4A). Therefore, by means of luciferase reporter assay we were able to validate the MXD1-miR-382-5p interaction (Figure 4B). Moreover, as shown in figure 4C, MXD1 protein level is strongly reduced in miR-382-5poverexpressing cells compared to control.

MXD1, also known as MAD1, is a transcriptional repressor involved in the MYC/MAX/MAD pathway, one of the best known network that controls cell proliferation and differentiation (23). In fact, MXD1 interacts with MAX antagonizing MYC/MAX dimer activity (23). Queva et al. showed that MXD1 is rapidly induced upon cell differentiation, when MYC expression is downregulated and cells begin to exit the cell cycle acquiring a differentiated phenotype (24).

Here we demonstrated that MXD1 knockdown reproduces the effects of miR-382-5p overexpression on CD34+ HSPC differentiation leading to a significant decrease of 
megakaryocyte precursors coupled to increase of granulocyte ones (Figure $\mathbf{5}$ and 6). According to our results, the increased expression of MXD1 has been previously reported also in megakaryocytes obtained in vitro from human CD34+ cells (21). Therefore, the MXD1 expression in CD34+ cells seems to be essential for megakaryocyte differentiation; in fact, the MK transcription factors GATA2, FLI1 and RUNX1 were significantly decreased after MXD1 silencing (Figure 7) (18), suggesting that the expression of these genes could be regulated by MXD1 in a direct or indirect way. For instance, the downregulation of GATA2, FLI1 and RUNX1 might be mediated by the MXD1-driven inhibition of a transcriptional repressor (i.e. BCL6) (25). Moreover, MXD1 can also heterodimerize with other proteins in addition to MAX (i.e. MLX), even if the function of these complexes as well as their targets has not been defined so far $(26,27)$.

Two independent studies reported that MXD1 induces erythroid differentiation in human (28) and mouse (29) erithroleukemia cells. Here we showed that MXD1 silencing did not impair erythroid differentiation in normal CD34+ cells, as demonstrated by the expression level of GPA (Figure S3, Panel C-D) and the percentage of red colonies (Figure 6A), which did not change after MXD1 knockdown. A possible explanation for this different cellular behaviour is that normal CD34+ cells might express some proteins that could replace MXD1 during erythroid differentiation.

Furthermore, our data demonstrated that MXD1 plays an important role in granulocyte differentiation; in fact, the MXD1 knockdown enhances the fraction of granulocyte precursors both in liquid and semisolid cultures (Figure 5 and 6). In agreement with our data, Foley et al. demonstrated that MXD1 knockout in mice affects the granulocyte lineage by reducing cell cycle withdrawal during the late stage 
of granulocyte differentiation, resulting in increased frequency of granulocytic precursors in the bone marrow (20). Moreover, another mechanism by which MXD1 inhibition could favour the expansion of granulocyte fraction is mediated by the reduction of MAX/MXD1 heterodimers arising in an increased availability of MAX protein, which in turn activates C/EBPalpha, a master regulator of granulocyte commitment (30).

Overall, these results demonstrated that miR-382-5p favours the expansion of granulocyte precursors through MXD1 down-regulation. Noteworthy, two independent research groups reported an high level of miR-382-5p in Acute promyelocytic leukemia (APL) patients with $t(15 ; 17)(10,11)$. This subtype of $A M L$ is characterized by PML-RARalpha fusion oncoprotein that is responsible for the differentiation block of leukemic promyelocytes. Of note, as demonstrated by Khan et al., PML-RARalpha can interact with MXD1 and inhibits its transcriptional repression activity (31). This evidence suggests that the deregulation of miR-382-5p/MXD1 axis contributes to the development of APL in cooperation with PML-RARalpha fusion protein; therefore, miR-382-5p could be considered as a novel molecular target for APL treatment.

In summary, our results demonstrated that miR-382-5p supports the expansion of granulocyte lineage whereas negatively interferes with megakaryocyte one through MXD1 down-regulation. To our knowledge, this is the first study describing miR-382$5 \mathrm{p} / \mathrm{MXD1}$ axis as a crucial regulator of hematopoietic stem cell fate.

\section{ACKNOWLEDGEMENT}

This work was supported by Associazione Italiana per la Ricerca sul Cancro (AIRC), project number \#10005 "Special Program Molecular Clinical Oncology 5x1000" to 
AGIMM (AIRC-Gruppo Italiano Malattie Mieloproliferative, http://www.progettoagimm.it); AIRC project number \# 15337; Italian Ministry of Health (Progetti di ricerca giovani ricercatori, Ricerca Finalizzata 2011-2012) project number \#GR-2011-02352109).

\section{AUTHOR DISCLOSURE STATEMENTS}

No competing financial interests exist.

\section{REFERENCES}

1. Shivdasani, R. A. (2006) MicroRNAs: regulators of gene expression and cell differentiation. Blood 108, 3646-3653

2. Olena, A. F., and Patton, J. G. (2010) Genomic organization of microRNAs. Journal of cellular physiology 222, 540-545

3. Gurtan, A. M., and Sharp, P. A. (2013) The role of miRNAs in regulating gene expression networks. Journal of molecular biology 425, 3582-3600

4. Arora, S., Rana, R., Chhabra, A., Jaiswal, A., and Rani, V. (2013) miRNAtranscription factor interactions: a combinatorial regulation of gene expression. Molecular genetics and genomics : MGG 288, 77-87

5. Lin, S., and Gregory, R. I. (2015) MicroRNA biogenesis pathways in cancer. Nature reviews. Cancer 15, 321-333

6. de Cubas, A. A., Leandro-Garcia, L. J., Schiavi, F., Mancikova, V., CominoMendez, I., Inglada-Perez, L., Perez-Martinez, M., Ibarz, N., Ximenez-Embun, P., Lopez-Jimenez, E., Maliszewska, A., Leton, R., Gomez Grana, A., Bernal, C., Alvarez-Escola, C., Rodriguez-Antona, C., Opocher, G., Munoz, J., Megias, D., Cascon, A., and Robledo, M. (2013) Integrative analysis of miRNA 
and mRNA expression profiles in pheochromocytoma and paraganglioma identifies genotype-specific markers and potentially regulated pathways. Endocrine-related cancer 20, 477-493

7. Chen, G., Han, N., Li, G., Li, X., Li, G., Li, Z., and Li, Q. (2016) Time course analysis based on gene expression profile and identification of target molecules for colorectal cancer. Cancer cell international 16, 22

8. Tan, H., He, Q., Gong, G., Wang, Y., Li, J., Wang, J., Zhu, D., and Wu, X. (2016) miR-382 inhibits migration and invasion by targeting ROR1 through regulating EMT in ovarian cancer. International journal of oncology 48, 181190

9. Qi, B., Lu, J. G., Yao, W. J., Chang, T. M., Qin, X. G., Ji, Y. H., Wang, T. Y., Liu, S. G., Li, H. C., Liu, Y. Z., and Zhao, B. S. (2015) Downregulation of microRNA-382 is associated with poor outcome of esophageal squamous cell carcinoma. World journal of gastroenterology 21, 6884-6891

10. Li, Z., Lu, J., Sun, M., Mi, S., Zhang, H., Luo, R. T., Chen, P., Wang, Y., Yan, M., Qian, Z., Neilly, M. B., Jin, J., Zhang, Y., Bohlander, S. K., Zhang, D. E., Larson, R. A., Le Beau, M. M., Thirman, M. J., Golub, T. R., Rowley, J. D., and Chen, J. (2008) Distinct microRNA expression profiles in acute myeloid leukemia with common translocations. Proceedings of the National Academy of Sciences of the United States of America 105, 15535-15540

11. Jongen-Lavrencic, M., Sun, S. M., Dijkstra, M. K., Valk, P. J., and Lowenberg, B. (2008) MicroRNA expression profiling in relation to the genetic heterogeneity of acute myeloid leukemia. Blood 111, 5078-5085

12. Norfo, R., Zini, R., Pennucci, V., Bianchi, E., Salati, S., Guglielmelli, P., Bogani, C., Fanelli, T., Mannarelli, C., Rosti, V., Pietra, D., Salmoiraghi, S., 
Bisognin, A., Ruberti, S., Rontauroli, S., Sacchi, G., Prudente, Z., Barosi, G., Cazzola, M., Rambaldi, A., Bortoluzzi, S., Ferrari, S., Tagliafico, E., Vannucchi, A. M., Manfredini, R., and Associazione Italiana per la Ricerca sul Cancro Gruppo Italiano Malattie Mieloproliferative, I. (2014) miRNA-mRNA integrative analysis in primary myelofibrosis CD34+ cells: role of miR155/JARID2 axis in abnormal megakaryopoiesis. Blood 124, e21-32

13. Bianchi, E., Zini, R., Salati, S., Tenedini, E., Norfo, R., Tagliafico, E., Manfredini, R., and Ferrari, S. (2010) c-myb supports erythropoiesis through the transactivation of KLF1 and LMO2 expression. Blood 116, e99-110

14. Humpe, A., Beck, C., Schoch, R., Kneba, M., and Horst, H. A. (2005) Establishment and optimization of a flow cytometric method for evaluation of viability of CD34+ cells after cryopreservation and comparison with trypan blue exclusion staining. Transfusion 45, 1208-1213

15. Salati, S., Zini, R., Bianchi, E., Testa, A., Mavilio, F., Manfredini, R., and Ferrari, S. (2008) Role of CD34 antigen in myeloid differentiation of human hematopoietic progenitor cells. Stem cells 26, 950-959

16. Zini, R., Norfo, R., Ferrari, F., Bianchi, E., Salati, S., Pennucci, V., Sacchi, G., Carboni, C., Ceccherelli, G. B., Tagliafico, E., Ferrari, S., Manfredini, R., and Investigators, A. (2012) Valproic acid triggers erythro/megakaryocyte lineage decision through induction of GFI1B and MLLT3 expression. Experimental hematology 40, 1043-1054 e1046

17. Guglielmelli, P., Zini, R., Bogani, C., Salati, S., Pancrazzi, A., Bianchi, E., Mannelli, F., Ferrari, S., Le Bousse-Kerdiles, M. C., Bosi, A., Barosi, G., Migliaccio, A. R., Manfredini, R., and Vannucchi, A. M. (2007) Molecular profiling of CD34+ cells in idiopathic myelofibrosis identifies a set of disease- 
associated genes and reveals the clinical significance of Wilms' tumor gene 1 (WT1). Stem cells 25, 165-173

18. Tijssen, M. R., Cvejic, A., Joshi, A., Hannah, R. L., Ferreira, R., Forrai, A., Bellissimo, D. C., Oram, S. H., Smethurst, P. A., Wilson, N. K., Wang, X., Ottersbach, K., Stemple, D. L., Green, A. R., Ouwehand, W. H., and Gottgens, B. (2011) Genome-wide analysis of simultaneous GATA1/2, RUNX1, FLI1, and SCL binding in megakaryocytes identifies hematopoietic regulators. Developmental cell 20, 597-609

19. Bianchi, E., Bulgarelli, J., Ruberti, S., Rontauroli, S., Sacchi, G., Norfo, R., Pennucci, V., Zini, R., Salati, S., Prudente, Z., Ferrari, S., and Manfredini, R. (2015) MYB controls erythroid versus megakaryocyte lineage fate decision through the miR-486-3p-mediated downregulation of MAF. Cell death and differentiation 22, 1906-1921

20. Foley, K. P., McArthur, G. A., Queva, C., Hurlin, P. J., Soriano, P., and Eisenman, R. N. (1998) Targeted disruption of the MYC antagonist MAD1 inhibits cell cycle exit during granulocyte differentiation. The EMBO journal 17, 774-785

21. Fuhrken, P. G., Chen, C., Apostolidis, P. A., Wang, M., Miller, W. M., and Papoutsakis, E. T. (2008) Gene Ontology-driven transcriptional analysis of CD34+ cell-initiated megakaryocytic cultures identifies new transcriptional regulators of megakaryopoiesis. Physiological genomics 33, 159-169

22. Borst, O., Schmidt, E. M., Munzer, P., Schonberger, T., Towhid, S. T., Elvers, M., Leibrock, C., Schmid, E., Eylenstein, A., Kuhl, D., May, A. E., Gawaz, M., and Lang, F. (2012) The serum- and glucocorticoid-inducible kinase 1 (SGK1) 
influences platelet calcium signaling and function by regulation of Orai1 expression in megakaryocytes. Blood 119, 251-261

23. Luscher, B. (2001) Function and regulation of the transcription factors of the Myc/Max/Mad network. Gene 277, 1-14

24. Queva, C., Hurlin, P. J., Foley, K. P., and Eisenman, R. N. (1998) Sequential expression of the MAD family of transcriptional repressors during differentiation and development. Oncogene 16, 967-977

25. Lee, S. C., Bottaro, A., Chen, L., and Insel, R. A. (2006) Mad1 is a transcriptional repressor of Bcl-6. Molecular immunology 43, 1965-1971

26. Billin, A. N., Eilers, A. L., Queva, C., and Ayer, D. E. (1999) Mlx, a novel Maxlike BHLHZip protein that interacts with the Max network of transcription factors. The Journal of biological chemistry 274, 36344-36350

27. Meroni, G., Cairo, S., Merla, G., Messali, S., Brent, R., Ballabio, A., and Reymond, A. (2000) Mlx, a new Max-like bHLHZip family member: the center stage of a novel transcription factors regulatory pathway? Oncogene 19, 32663277

28. Acosta, J. C., Ferrandiz, N., Bretones, G., Torrano, V., Blanco, R., Richard, C., O'Connell, B., Sedivy, J., Delgado, M. D., and Leon, J. (2008) Myc inhibits p27-induced erythroid differentiation of leukemia cells by repressing erythroid master genes without reversing p27-mediated cell cycle arrest. Molecular and cellular biology $28,7286-7295$

29. Cultraro, C. M., Bino, T., and Segal, S. (1997) Function of the c-Myc antagonist Mad1 during a molecular switch from proliferation to differentiation. Molecular and cellular biology 17, 2353-2359 
30. Zada, A. A., Pulikkan, J. A., Bararia, D., Geletu, M., Trivedi, A. K., Balkhi, M. Y., Hiddemann, W. D., Tenen, D. G., Behre, H. M., and Behre, G. (2006) Proteomic discovery of Max as a novel interacting partner of C/EBPalpha: a Myc/Max/Mad link. Leukemia 20, 2137-2146

31. Khan, M. M., Nomura, T., Kim, H., Kaul, S. C., Wadhwa, R., Shinagawa, T., Ichikawa-Iwata, E., Zhong, S., Pandolfi, P. P., and Ishii, S. (2001) Role of PML and PML-RARalpha in Mad-mediated transcriptional repression. Molecular cell 7, 1233-1243

\section{FIGURE LEGENDS}




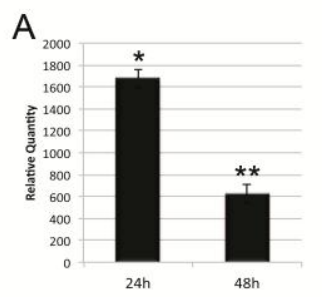

$\mathrm{B}_{90}$

$\mathrm{C}_{70}$
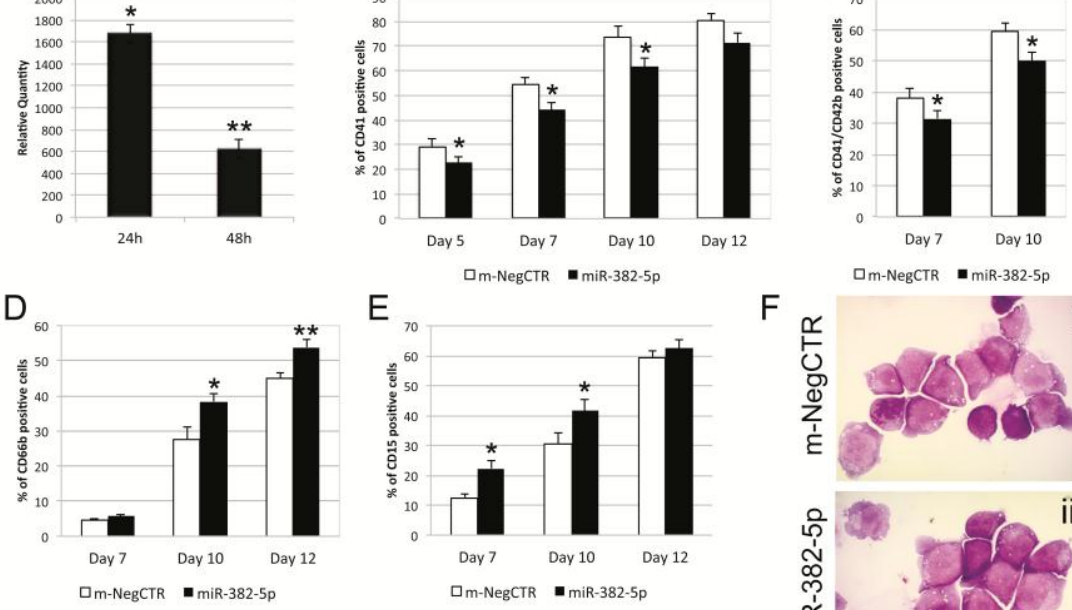

$E$

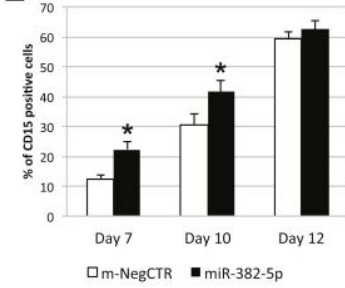

$\mathrm{F}$

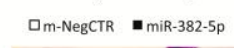

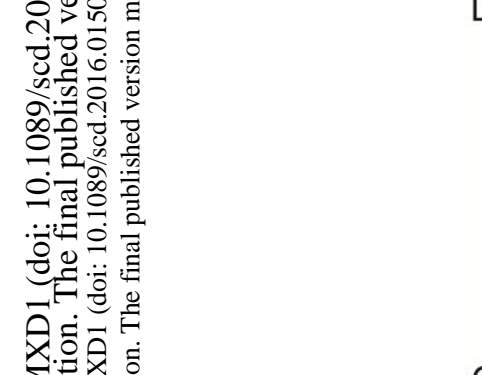

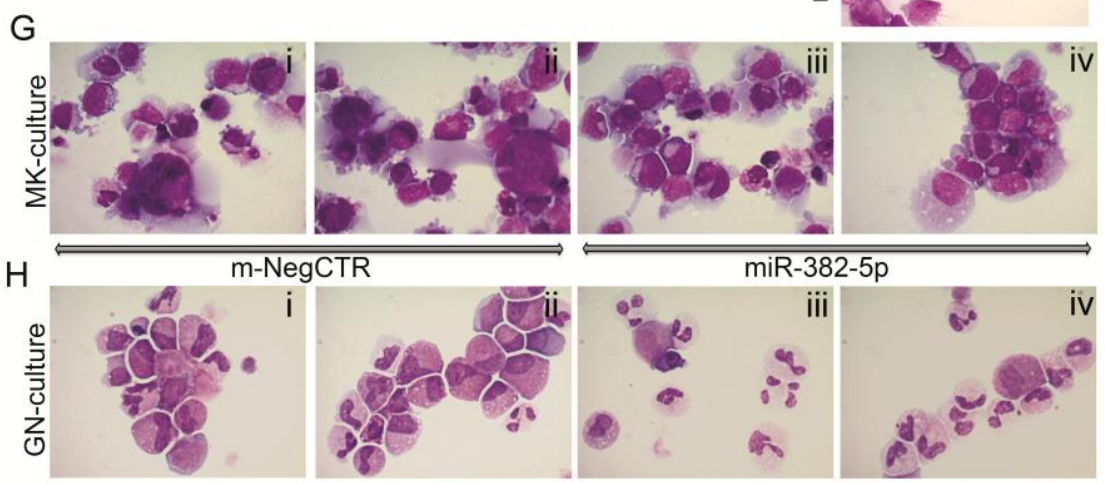

Figure 1

Figure 1. Effect of miR-382-5p overexpression on CD34+ HSPC differentiation.

(A) The expression level of miR-382-5p at 24 and 48 hours after the last nucleofection was measured by qRT-PCR and data reported as Relative Quantity. (B-C) Flow cytometry results of CD41, and CD42b expression at day 5, 7, 10, and 12 after the last nucleofection in MK unilineage culture. (D-E) Flow cytometry results of CD66b, and CD15 expression at day 7, 10, and 12 after the last nucleofection in GN unilineage culture. (F) Morphological analysis of m-NegCTR (i) and miR-382-5p (ii) nucleofected cells after May-Grünwald-Giemsa staining at 24 hours after the last nucleofection (before seeding in unilineage and multilineage cultures) in a representative experiment. Magnification $\times 1000$. (G) Morphological analysis of $\mathrm{m}$ NegCTR (i-ii) and miR-382-5p (iii-iv) nucleofected cells after May-Grünwald-Giemsa 
staining at days 10 of MK unilineage culture in a representative experiment. Magnification $\times 1000$. $(\mathrm{H})$ Morphological analysis of m-NegCTR (i-ii) and miR-382-5p (iii-iv) nucleofected cells after May-Grünwald-Giemsa staining at days 10 of GN unilineage culture in a representative experiment. Magnification $\times 1000$.

Values are reported as mean \pm standard error of the mean (SEM). ${ }^{* *}, P<.01$ versus m-NegCTR; * $P<.05$ versus $m-N e g C T R$. The results come from five independent experiments. Abbreviations: m-NegCTR, mimic negative control; $h$, hours. 


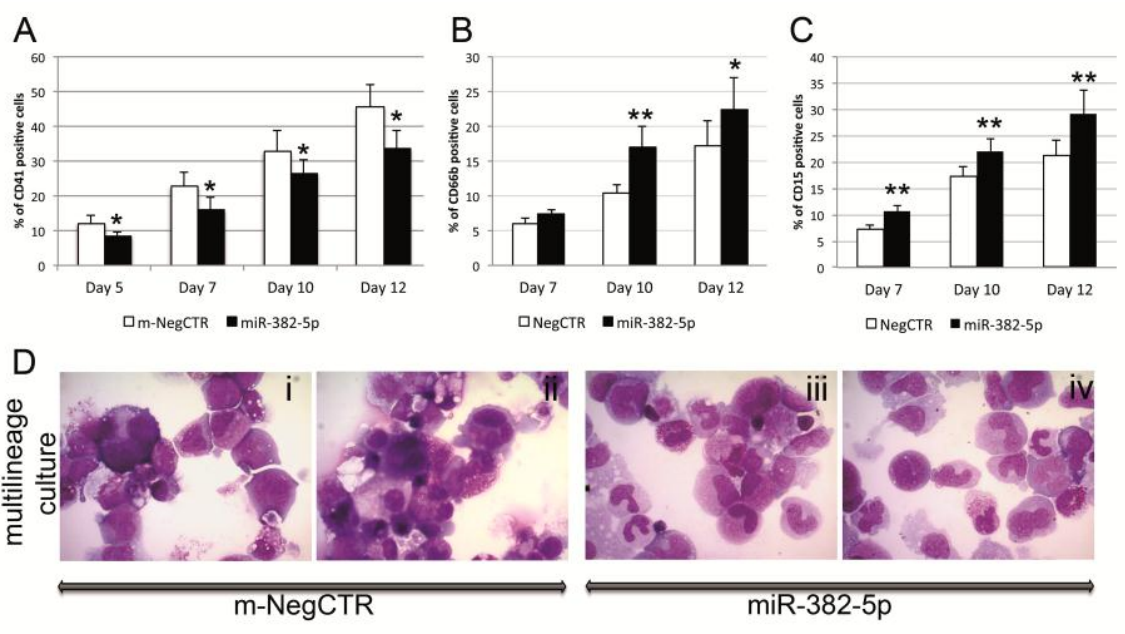

Figure 2

Figure 2. Effect of miR-382-5p overexpression on CD34+ HSPC differentiation in multilineage culture.

(A) Flow cytometry results of CD41 expression at day 5, 7, 10, and 12 after the last nucleofection in multilineage culture. (B-C) Flow cytometry results of CD66b, and CD15 expression at day 7, 10, and 12 after the last nucleofection in multilineage culture. (D) Morphological analysis of m-NegCTR (i-ii) and miR-382-5p (iii-iv) nucleofected cells after May-Grünwald-Giemsa staining at day 10 of multilineage culture in a representative experiment. Magnification $\times 1000$.

Values are reported as mean \pm standard error of the mean (SEM). ${ }^{* *}, P<.01$ versus m-NegCTR; ${ }^{*}, P<.05$ versus $m-N e g C T R$. The results come from five independent experiments. Abbreviations: m-NegCTR, mimic negative control. 


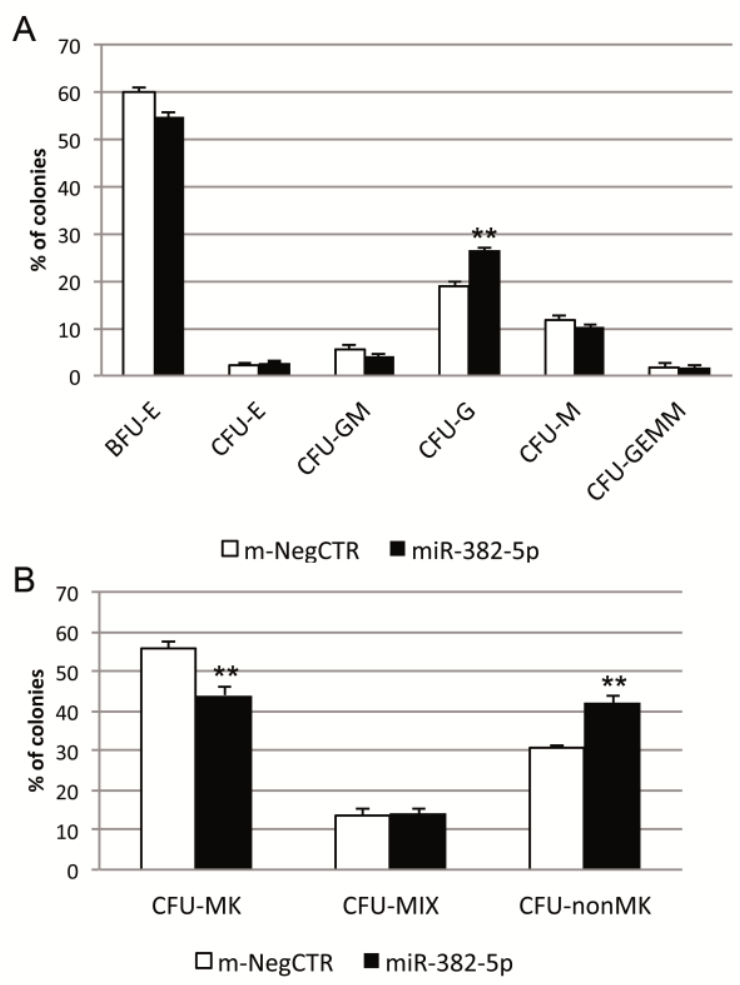

Figure 3

Figure 3. Methylcellulose and collagen-based clonogenic assays upon miR382-5p overexpression in CD34+ HSPCs.

(A) Results of the statistical analysis of methylcellulose clonogenic assay. The cells were plated 24 hours after the last nucleofection and scored after 14 days. (B) Results of the statistical analysis of collagen-based clonogenic assay. The cells were plated 24 hours after the last nucleofection and scored after 12 days.

Values are reported as mean \pm standard error of the mean (SEM). ${ }^{*}, P<.01$ versus m-NegCTR; ${ }^{*}, P<.05$ versus $m-N e g C T R$. The results come from five independent experiments. Abbreviations: CFU, colony-forming unit; BFU, burst-forming units; MK, megakaryocyte; G, granulocyte; M, monocyte; E, erythroid; GM, granulo-monocyte. 


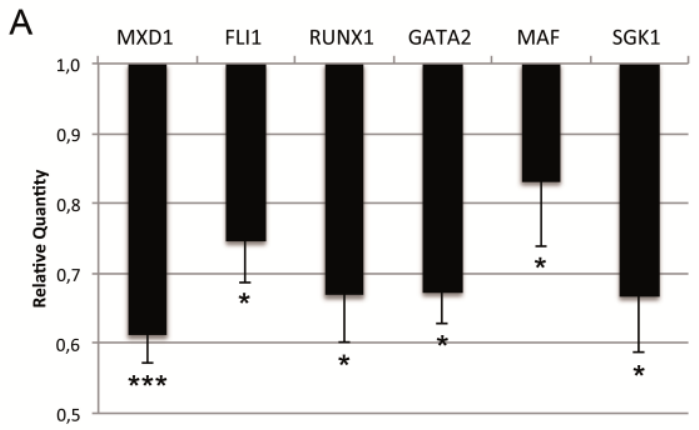

$\mathrm{B}$

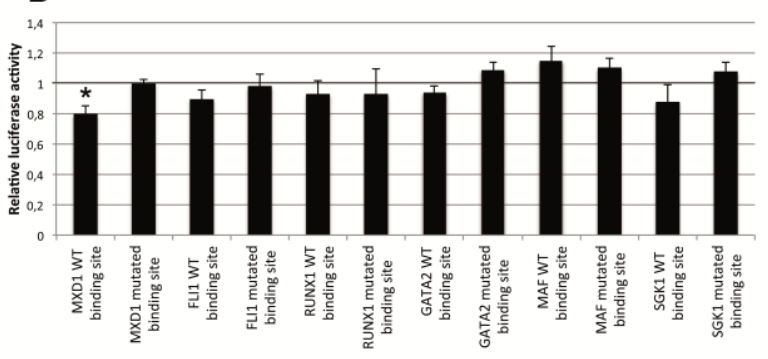

C

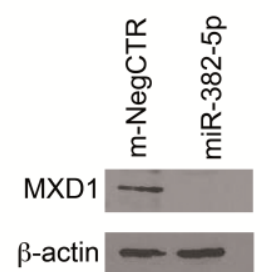

Figure 4

\section{Figure 4. Identification of miR-382-5p targets.}

(A) The expression levels of the putative targets were measured by qRT-PCR in miR382-5p-overexpressing CD34+ cells at 24 hours after the last nucleofection. Data are reported as Relative Quantity. Results come from five independent experiments. Values are reported as mean $\pm \mathrm{SEM} ;{ }^{* \star *}, P<.001$ versus $\mathrm{m}-\mathrm{NegCTR}{ }^{* *}, P<.01$ versus m-NegCTR; *, $P<.05$ versus m-NegCTR. (B) Results of the luciferase reporter assays performed with wild type and mutated miRNA binding sites. Normalized luciferase activity of K562 cells nucleofected with miR-382-5p mimics and pmirGLO vectors containing the putative miRNA binding sites. Firefly luciferase activity was measured 24 hours after the nucleofection and normalized to Renilla luciferase activity. Each bar represents the luciferase activity upon miRNA overexpression normalized on the value of the same 3'-UTR luciferase vector upon 
m-NegCTR transfection. (C) Western blot analysis of MXD1 protein levels in lysates from miR-382-5p and m-NegCTR transfected CD34+ cells at 24 hours after the last nucleofection. $\beta$-actin protein level is reported as loading control.

Values are reported as mean $\pm \mathrm{SEM} ;{ }^{*}, P<.05$ versus mutated miRNA binding site. Results come from three independent experiments performed in duplicate. 

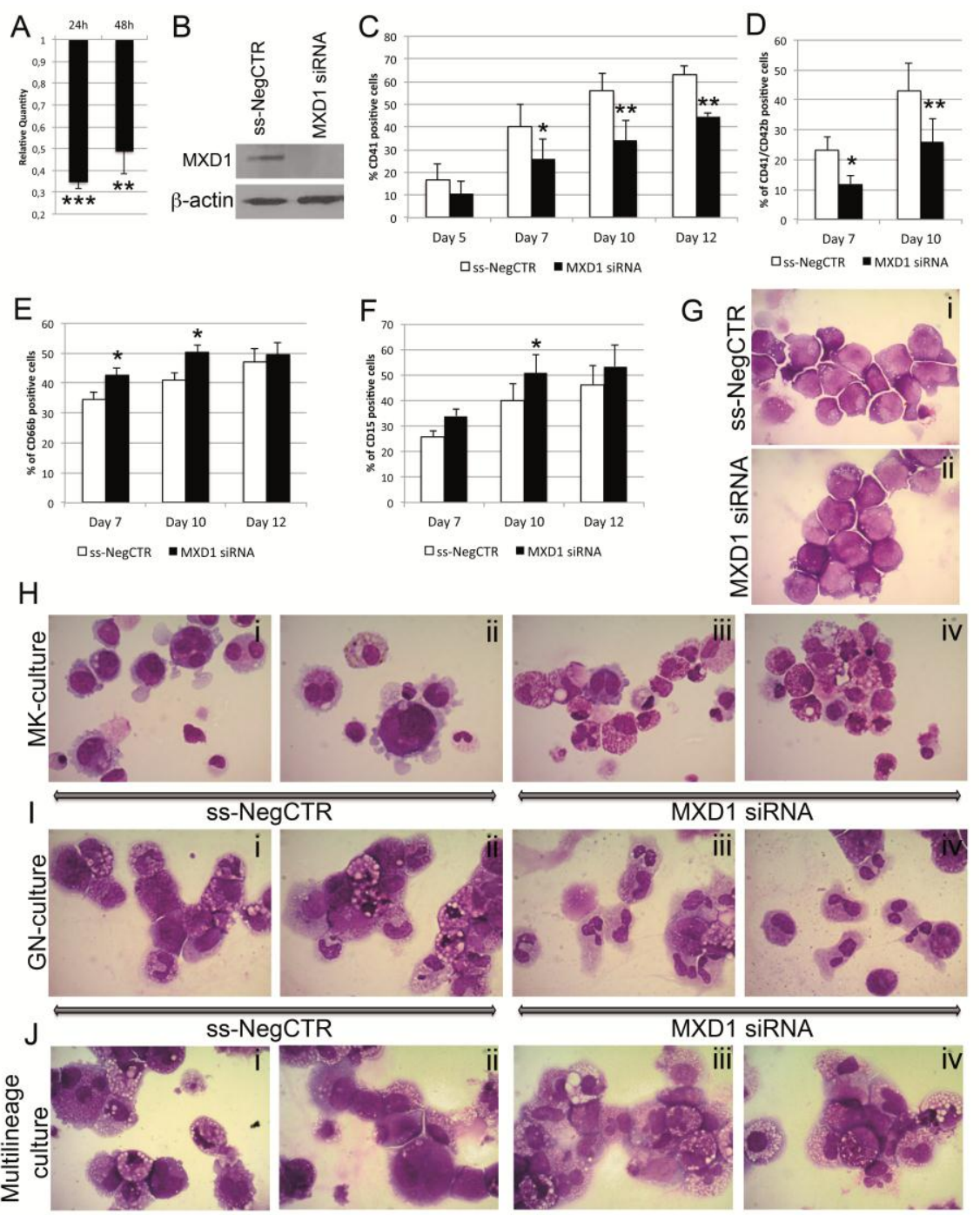

Figure 5

Figure 5. Effect of MXD1 silencing on CD34+ HSPC differentiation.

(A) The expression level of MXD1 at 24 and 48 hours after the last nucleofection was measured by qRT-PCR and data reported as Relative Quantity. (B) Western blot analysis of MXD1 protein level in lysates from MXD1 siRNA and ss-NegCTR transfected CD34+ cells at 24 hours after the last nucleofection. $\beta$-actin protein level is reported as loading control. (C-D) Flow cytometry results of CD41, and CD42b expression at day 5, 7, 10, and 12 after the last nucleofection in MK unilineage culture. (E-F) Flow cytometry results of CD66b, and CD15 expression at day 7, 10, and 12 after the last nucleofection in GN unilineage culture. (G) Morphological 
analysis of negative control (ss-NegCTR) (i) and MXD1 short interfering RNA (MXD1 siRNA) (ii) samples after May-Grünwald-Giemsa staining at 24 hours after the last nucleofection (before seeding in unilineage and multilineage cultures) in a representative experiment. Magnification $\times 1000$. (H) Morphological analysis of negative control (ss-NegCTR) (i-ii) and MXD1 short interfering RNA (MXD1 siRNA) (iii-iv) samples after May-Grünwald-Giemsa staining at day 10 of MK unilineage culture in a representative experiment. Magnification $\times 1000$. (I) Morphological analysis of ss-NegCTR (i-ii) and MXD1 siRNA (iii-iv) samples after May-GrünwaldGiemsa staining at day 10 of GN unilineage culture in a representative experiment. Magnification $\times 1000$. (J) Morphological analysis of ss-NegCTR (i-ii) and MXD1 siRNA (iii-iv) samples after May-Grünwald-Giemsa staining at day 10 of multilineage culture in a representative experiment. Magnification $\times 1000$.

Values are reported as mean \pm standard error of the mean (SEM). ${ }^{* *}, P<.001$ versus ss-NegCTR; ${ }^{* *}, \mathrm{P}<.01$ versus ss-NegCTR; ${ }^{*}, \mathrm{P}<.05$ versus ss-NegCTR. The results come from five independent experiments. Abbreviations: ss-NegCTR, siRNA negative control; siRNA, small interfering RNA; $h$, hours. 

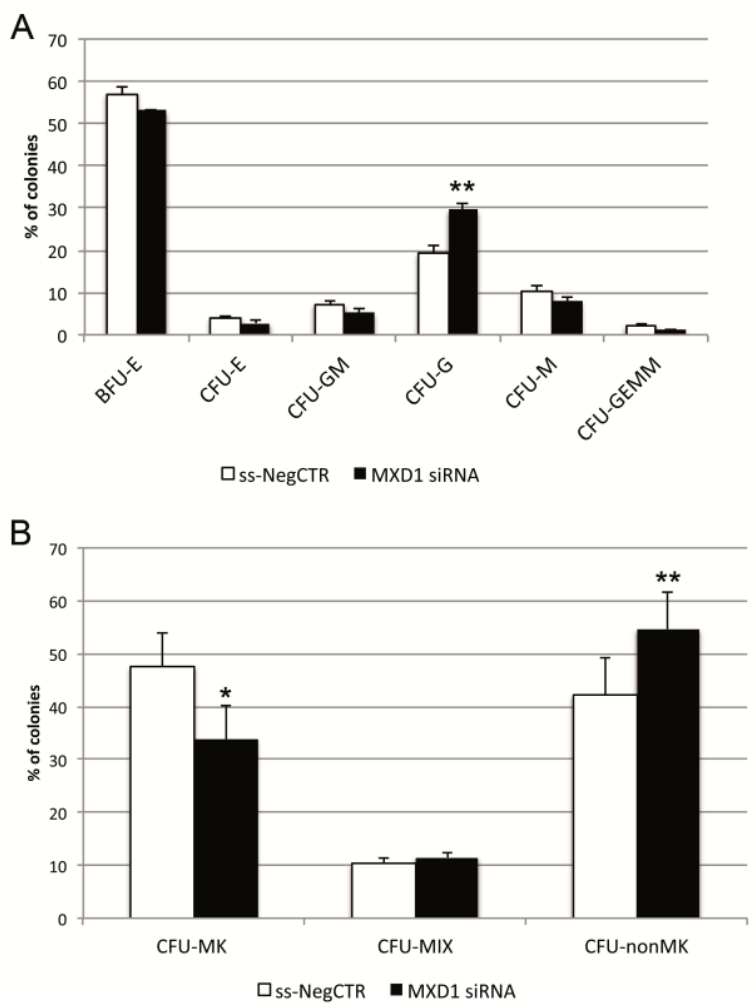

Figure 6

Figure 6. Methylcellulose and collagen-based clonogenic assays upon MXD1 silencing in CD34+ HSPCs.

(A) Results of the statistical analysis of methylcellulose clonogenic assay. The cells were plated 24 hours after the last nucleofection and scored after 14 days. (B) Results of the statistical analysis of collagen-based clonogenic assay. The cells were plated 24 hours after the last nucleofection and scored after 12 days.

Values are reported as mean \pm standard error of the mean (SEM). ${ }^{*}, \mathrm{P}<.01$ versus ss-NegCTR; ${ }^{*}, \mathrm{P}<.05$ versus ss-NegCTR. The results come from five independent experiments. Abbreviations: CFU, colony-forming unit; BFU, burst-forming units; MK, megakaryocyte; G, granulocyte; M, monocyte; E, erythroid; GM, granulo-monocyte. 


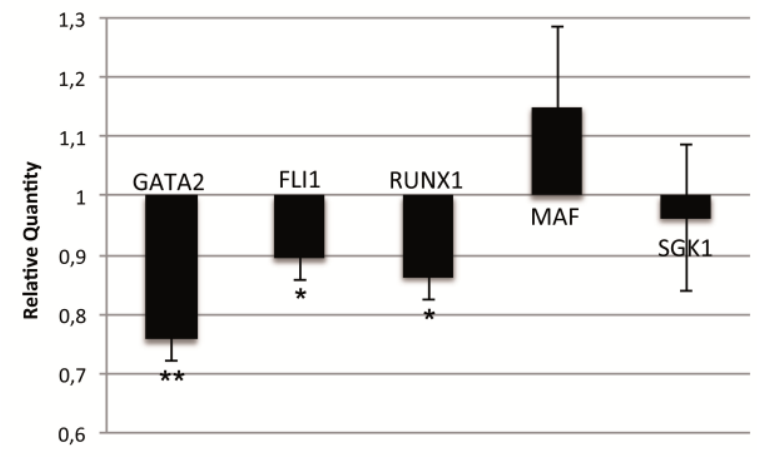

Figure 7

Figure 7. MXD1 downstream regulation of GATA2, FLI1 and RUNX1.

The expression levels of GATA2, FLI1, RUNX1, MAF, and SGK1 were measured by qRT-PCR in MXD1 siRNA-transfected CD34+ cells at 24 hours after the last nucleofection. Data are reported as Relative Quantity. Values are reported as mean \pm standard error of the mean (SEM). The results come from five independent experiments. ${ }^{* *}, \mathrm{P}<.01$ versus ss-NegCTR; ${ }^{*}, \mathrm{P}<.05$ versus ss-NegCTR. 


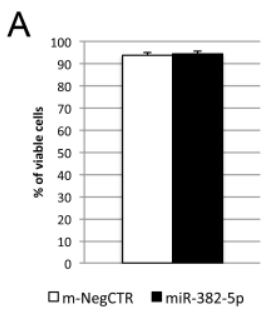

B
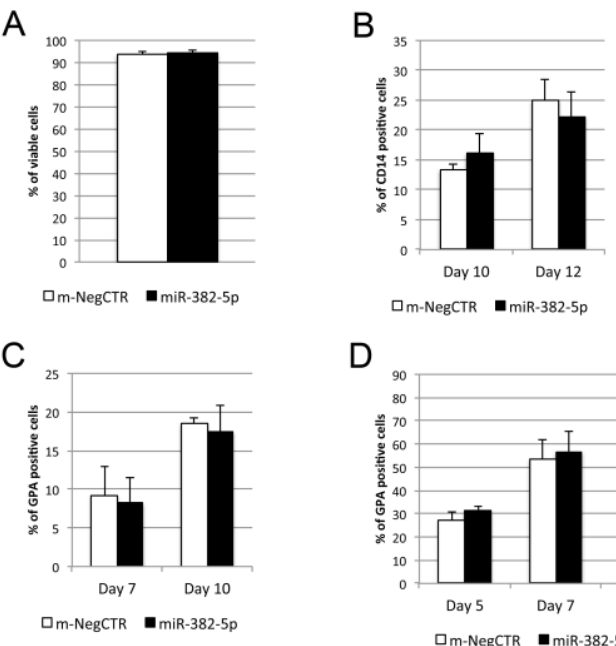

D

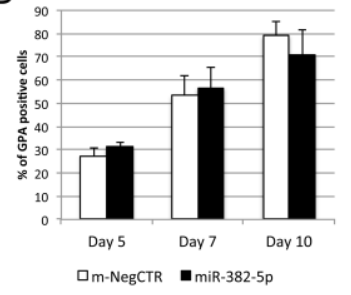

Figure S1

Figure S1. Effect of miR-382-5p overexpression in CD34+ HSPC on cell viability and differentiation of the erythroid and monocyte/macrophage lineages.

(A) Cell viability assessed by trypan blue exclusion assay in m-NegCTR or miR-382$5 p$ samples at 24 hours after the last nucleofection. (B) Expression of monocyte/macrophage marker CD14 at day 10, and 12 after the last nucleofection in multilineage culture. (C) Expression of erythrocyte marker GPA at day 7, and 10 after the last nucleofection in serum-free multilineage culture. (D) Expression of erythrocyte marker GPA at day 5, 7, and 10 after the last nucleofection in EPO unilineage culture.

Values are reported as mean \pm standard error of the mean (SEM). ${ }^{*}, P<.05$ versus m-NegCTR. The results come from three independent experiments. Abbreviations: m-NegCTR, mimic negative control. 

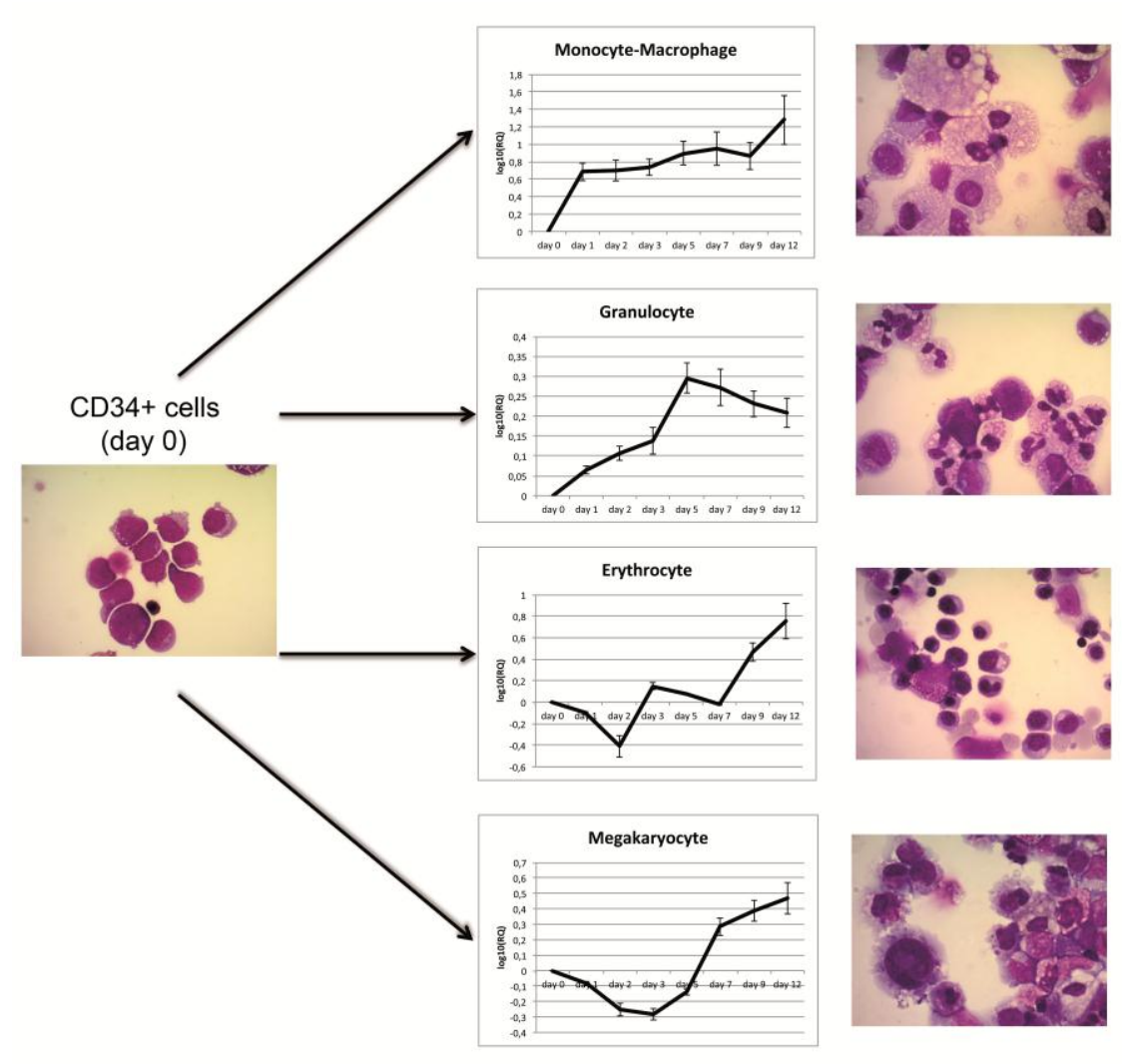

Figure S2

Figure S2. MiR-382-5p expression during erythroid, megakaryocyte, granulocyte and mono-macrophage differentiation.

After 24 hours of culture (day 0 ) the cells were seeded in erythrocyte, megakaryocyte, granulocyte and monocyte unilineage cultures. CD34+ cells differentiation was monitored by morphological analysis of MGG-stained cytospins at day 9. MiR-382-5p expression level was detected by qRT-PCR at different time points (i.e. days $1,2,3,5,7,9$ and 12) and data reported as $\log 10$ of the Relative Quantity (RQ). The day 0 sample was designated as the calibrator sample.

Values are reported as mean \pm standard error of the mean (SEM). The results come from two independent experiments. Abbreviations: Relative Quantity, RQ. 

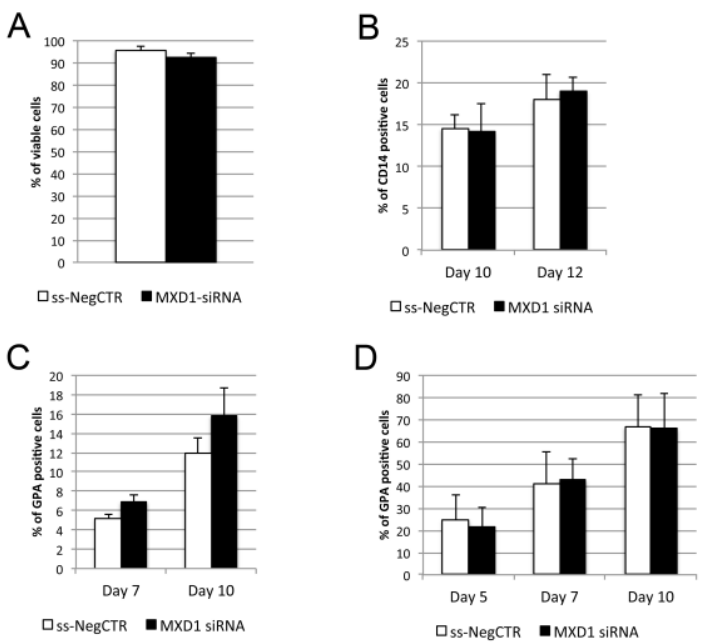

Figure S3

Figure S3. Effect of MXD1 silencing in CD34+ HSPC on cell viability and differentiation of the erythroid and monocyte/macrophage lineages.

(A) Cell viability assessed by trypan blue exclusion assay in ss-NegCTR or MXD1siRNA samples at 24 hours after the last nucleofection. (B) Expression of monocyte/macrophage marker CD14 at day 10 and 12 after the last nucleofection in multilineage culture. (C) Expression of erythrocyte marker GPA at day 7, and 10 after the last nucleofection in serum-free multilineage culture. (D) Expression of erythrocyte marker GPA at day 5, 7, and 10 after the last nucleofection in EPO unilineage culture.

Values are reported as mean \pm standard error of the mean (SEM). ${ }^{*}, \mathrm{P}<.05$ versus ss-NegCTR. The results come from three independent experiments. Abbreviations: ss-NegCTR, siRNA negative control; siRNA, small interfering RNA. 
Table 1. miRNA target prediction analysis

\begin{tabular}{cccccccc}
\hline $\begin{array}{l}\text { TARGET } \\
\text { GENE }\end{array}$ & RefSeq & $\begin{array}{c}\text { TargetScan } \\
\text { (total contex RG } \\
\text { ++ score) }\end{array}$ & $\begin{array}{c}\text { microRNA.O } \\
\text { score) }\end{array}$ & $\begin{array}{c}\text { PITA } \\
\text { (score) }\end{array}$ & $\begin{array}{c}\text { Diana } \\
\text { microT } \\
\text { (miTG } \\
\text { score) }\end{array}$ & BCmicro & $\begin{array}{c}\text { RNA22 } \\
\text { (pvalue) }\end{array}$ \\
FLI1 & NM_002017 & -0.02 & NULL & -8.43 & NULL & 0,02359801 & NULL \\
GATA2 & NM_032638 & -0.02 & -0.2130 & -3.5 & 0.506 & 0,04337399 & $7.63 E-3$ \\
MAF & NM_005360 & -0.01 & NULL & NULL & 0.494 & 0,00117862 & $4.5 E-2$ \\
MXD1 & NM_002357 & -0.09 & -0.3933 & -7.5 & 0.634 & 0,34630233 & $5.06 E-3$ \\
RUNX1 & NM_001754 & -0.02 & -0.7503 & -5.03 & 0.615 & 0,23419901 & $2.21 E-1$ \\
SGK1 & NM_005627 & -0.12 & -0.7717 & -9.51 & NULL & 0,85408936 & NULL \\
\hline & & & & & & & \\
\hline
\end{tabular}




\begin{tabular}{|c|c|c|c|c|c|c|c|}
\hline 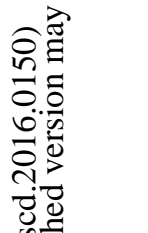 & \multicolumn{7}{|c|}{ Table S1. Sequences of siRNA targeting Mxd1 mRNA } \\
\hline 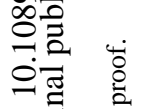 & $\begin{array}{l}\text { RefSeq Accession } \\
\text { Number }\end{array}$ & Gene Symbol & Full Gene Name & Gene ID & SiRNA ID & Sense siRNA Sequence & Antisense siRNA Sequence \\
\hline 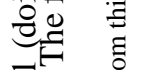 & NM_002357 & MXD1 & MAX dimerization protein 1 & 4084 & s8388 & GCAGAUCAACUCACAAUGAtt & UCAUUGUGAGUUGAUCUGCta \\
\hline & NM_002357 & MXD1 & MAX dimerization protein 1 & 4084 & s8389 & CCAUGUUACCAUACAAUAAtt & UUAUUGUAUGGUAACAUGGag \\
\hline 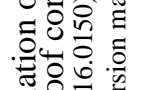 & NM_002357 & MXD1 & MAX dimerization protein 1 & 4084 & s8390 & GCAUCAAGAGAAUAAAGCUtt & AGCUUUAUUCUCUUGAUGCtg \\
\hline
\end{tabular}


Table S2. The list of luciferase reporter constructs

\begin{tabular}{|c|c|c|c|}
\hline GENE SYMBOL & VECTOR & Representative transcript & ANNEALING OLIGONUCLEOTIDES \\
\hline I & pmirGLO & 1 & 3'UTR-less \\
\hline \multirow[t]{2}{*}{ MXD1 } & \multirow[t]{2}{*}{ pmirGLO } & \multirow[t]{2}{*}{ ENST00000264444.2 } & $\begin{array}{l}\text { wild type: 5'AAACTAGCGGCCGCTAGTGATTTGCACTGAAATACAACTTGT3' } \\
\text { 3'TTTGATCGCCGGCGATCACTAAACGTGACTTTATGTTGAACAGATC5' }\end{array}$ \\
\hline & & & $\begin{array}{l}\text { mutant: 5'AAACTAGCGGCCGCTAGTGATTTGCgCTGAAATACAgtcTGT3' } \\
\text { 3'TTTGATCGCCGGCGATCACTAAACGcGACTTTATGTcagACAGATC5' }\end{array}$ \\
\hline \multirow[t]{2}{*}{ FLI1 } & \multirow[t]{2}{*}{ pmirGLO } & \multirow[t]{2}{*}{ ENST00000527786.2 } & $\begin{array}{l}\text { wild type: 5'AAACTAGCGGCCGCTAGTCAACCAATCAGAAGGCAACTTACT3' } \\
\text { 3'TTTGATCGCCGGCGATCAGTTGGTTAGTCTTCCGTTGAATGAGATC5' }\end{array}$ \\
\hline & & & $\begin{array}{l}\text { mutant: 5'AAACTAGCGGCCGCTAGTCAACCAATCAGAAGGCAAtccACT3' } \\
\text { 3'TTTGATCGCCGGCGATCAGTTGGTTAGTCTTCCGTTaggTGAGATC5' }\end{array}$ \\
\hline \multirow[t]{2}{*}{ RUNX1 } & \multirow[t]{2}{*}{ pmirGLO } & \multirow[t]{2}{*}{ ENST00000344691.4 } & $\begin{array}{l}\text { wild type: 5'AAACTAGCGGCCGCTAGTCTGGACTTATTTAAAACAACTTGT3' } \\
\text { 3'TTTGATCGCCGGCGATCAGACCTGAATAAATTTTGTTGAACAGATC5' }\end{array}$ \\
\hline & & & $\begin{array}{l}\text { mutant: 5'AAACTAGCGGCCGCTAGTCTGGACTTATTTAAAACAgtcTGT3' } \\
\text { 3'TTTGATCGCCGGCGATCAGACCTGAATAAATTTTGTCagACAGATC5' }\end{array}$ \\
\hline \multirow[t]{2}{*}{ GATA2 } & \multirow[t]{2}{*}{ pmirGLO } & \multirow[t]{2}{*}{ ENST00000341105.2 } & $\begin{array}{l}\text { wild type: 5'AAACTAGCGGCCGCTAGTTGGAGAACAACAAGGACAACTTTT3' } \\
\text { 3'TTTGATCGCCGGCGATCAACCTCTTGTTGTTCCTGTTGAAAAGATC5' }\end{array}$ \\
\hline & & & $\begin{array}{l}\text { mutant: 5'AAACTAGCGGCCGCTAGTTGGAGAACAACAAGGACAgtcTTT3' } \\
\text { 3'TTTGATCGCCGGCGATCAACCTCTTGTTGTTCCTGTcagAAAGATC5' }\end{array}$ \\
\hline \multirow[t]{2}{*}{ SGK1 } & \multirow[t]{2}{*}{ pmirGLO } & \multirow[t]{2}{*}{ ENST00000367858.5 } & $\begin{array}{l}\text { wild type: 5'AAACTAGCGGCCGCTAGTCCCTCATCCCATCACACAACTTTTT' } \\
\text { 3'TTTGATCGCCGGCGATCAGGGAGTAGGGTAGTGTGTTGAAAAGATC5' }\end{array}$ \\
\hline & & & $\begin{array}{l}\text { mutant: 5'AAACTAGCGGCCGCTAGTCCCTCATCCCATCACACAgtcTTT3' } \\
\text { 3'TTTGATCGCCGGCGATCAGGGAGTAGGGTAGTGTGTcagAAAGATC5' }\end{array}$ \\
\hline \multirow[t]{2}{*}{ MAF } & \multirow[t]{2}{*}{ pmirGLO } & \multirow[t]{2}{*}{ ENST00000393350.1 } & $\begin{array}{l}\text { wild type: 5'AAACTAGCGGCCGCTAGTTACAGCATGTGCTTGCAACTTAAT3' } \\
\text { 3'TTTGATCGCCGGCGATCAATGTCGTACACGAACGTTGAATTAGATC5' }\end{array}$ \\
\hline & & & $\begin{array}{l}\text { mutant: 5'AAACTAGCGGCCGCTAGTTACAGCATGTGCTTGCAAtccAAT3' } \\
\text { 3'TTTGATCGCCGGCGATCAATGTCGTACACGAACGTTaggTTAGATC5' }\end{array}$ \\
\hline
\end{tabular}

